\title{
Contribución de Graells a la posición de España en el primer convenio internacional para la protección de ciertas especies de la fauna silvestre (1902)
}

\author{
J. J. Ferrero-García*
}

El 19 de marzo de 1902 doce naciones europeas firmaron en París el Convenio Internacional para la Protección de las Aves Útiles a la Agricultura (en adelante el Convenio), que permaneció vigente hasta 1950 (van Heijnsbergen, 1997; Boardman, 2006). Este tratado constituye la primera norma internacional para la defensa de la vida silvestre jurídicamente vinculante, pues la convención que habían suscrito diversos países dos años antes, en Londres, para la preservación de determinadas especies animales de África, nunca llegó a entrar en vigor (Kiss \& Shelton, 2004; Bowman et al., 2010). A pesar de que muchos autores han reprobado el utilitarismo y la visión antropocéntrica del Convenio (e.g., Bowman \& Redgwell, 1996; Desai, 2010), conviene también recordar su carácter innovador en bastantes aspectos, especialmente por la salvaguardia absoluta que ofrecía a unos 150 taxones (Bowman et al., 2010), la protección de los huevos y los nidos de todas las aves, o la prohibición del uso de los métodos masivos y no selectivos de captura (Sands, 1995).

De una u otra manera, el Convenio no fue un acto jurídico improvisado, sino el fruto de varias iniciativas supranacionales (van Heijnsbergen, 1997). Estas comenzaron en 1868, se encauzaron en sucesivos acontecimientos destacables, como el Primer Congreso Ornitológico Internacional, celebrado en Viena en 1884 (Oustalet, 1884), y desembocaron en una conferencia específica organizada por Francia, en junio de 1895. En esta importante reunión, naturalistas de prestigio, técnicos y políticos consensuaron un borrador o proyecto de Convenio que, después de su ulterior análisis por los gobiernos de los dieciséis Estados participantes, se firmaría con algunas modificaciones por solo doce de ellos, siete años más tarde en París (Herman, 1907). Asimismo, debe tenerse en cuenta que la división de los animales en «útiles»y «perjudiciales» era una idea imperante en la comunidad científica de la época (e.g., Vogt, 1867; GarcíaMaceira, 1882; Graells, 1882; Herman \& Owen, 1909), y que apenas fue cuestionada por la mayoría de los eminentes zoólogos (el francés E. Oustalet, el italiano E. Giglioli, el británico H. Saunders, el suizo V. Fatio, el austro-húngaro V. von Tschusi, etc.) que asistieron a la conferencia de 1895 (Herman, 1907). De hecho, en aquellos años el debate se centraba, más que en la idoneidad de dicha clasificación, en la presunta utilidad o perjuicio de numerosas especies en particular (Herman, 1907, Housse, 1929; Torres-Vila et al., 2009). En cualquier caso, la preocupación que surgió en el siglo XIX por el futuro de las aves ha sido destacada reiteradamente tanto en esa centuria como en las décadas posteriores (e.g., Michelet, 1858; Oustalet, 1884 y 1893; MacPherson \& Momber, 1909; Barclay-Smith, 1959).

Una de las doce naciones que suscribió el Convenio fue España (Ministerio de Estado, 1907; SEO, 1956), que por aquel entonces ya había elaborado su propia normativa conservacionista (Ministerio de Fomento, 1896; Peña, 1905), en consonancia con la existente en otros países europeos (MacPherson \& Momber, 1909). Esta ratificación supuso, al menos sobre el papel, el reforzamiento de la protección de muchas aves total o parcialmente insectívoras (Apodiformes, Passeriformes, Piciformes, Strigiformes, etc.), pero también el amparo de algunos taxones desprotegidos hasta entonces, en 
concreto de las cigüeñas (Ciconia ciconia y $C$. nigra) y del abejaruco europeo (Merops apiaster) (Ferrero-García, 2010). Como recientemente se ha puesto de relieve (Ferrero-García, 2011), el ilustre personaje que impulsó y diseñó en España estas medidas pioneras fue Mariano de la Paz Graells Agüera (1809-1898), en el marco de su actividad en el Consejo Superior de Agricultura, Industria y Comercio (en lo sucesivo CSA), un organismo público dependiente del Ministerio de Fomento. Esta faceta de Graells se une así a otras muchas ampliamente conocidas y que le sitúan como uno de los mayores naturalistas españoles del siglo XIX (Rábano \& Aragón, 2007; Izquierdo \& Martín, 2008; Casado, 2009; Cervantes, 2009; Aragón \& Villena, 2010).

El objetivo de este trabajo es demostrar que fue el CSA la institución que llevó a cabo, en España, el examen del borrador del Convenio elaborado en la conferencia de 1895 y el que dio su conformidad técnica; y fundamentalmente que en el seno del CSA el encargado de realizar esta tarea fue Graells, quien redactó ad hoc un exhaustivo informe favorable, aunque con unas interesantes puntualizaciones que revelan su posición personal sobre este asunto. Para lograr este propósito se ha investigado en el Archivo central del Ministerio de Agricultura, Alimentación y Medio Ambiente (área de Agricultura y Alimentación), situado en Madrid (en adelante AMA), donde se depositaron las actas del CSA tras la desaparición de esta entidad. Asimismo, se han consultado los documentos custodiados en el Archivo del Museo Nacional de Ciencias Naturales de Madrid (en adelante AMNCN), adscrito al Consejo Superior de Investigaciones Científicas (CSIC); concretamente, los correspondientes al Fondo Personal Científico, en parte dedicado a Graells, debido a la estrecha vinculación que hubo entre este y el Museo (Aragón, 2009; Izquierdo \& Martín, 2009). Todas las notas y expedientes del AMNCN que se citan pertenecen a la misma signatura (ACN0345/018) y, en los del AMA, sucede idéntica circunstancia (signatura: 238/B; carpeta 1; años: 1892-1901).

El relato de los hechos comienza cuando el embajador de Francia, el 6 de marzo de 1896, se dirige al Gobierno español remitiéndole formalmente el proyecto de Convenio y proponiéndole su plena adhesión (AMNCN; documento 18). Tras unos trámites previos a través del Ministerio de Estado, el ministro de Fomento A. Linares Rivas requirió, el
22 de junio de 1896, al presidente del CSA para que emitiera un informe acerca del referido proyecto (AMNCN; documento 19). A su vez, el presidente del CSA escribió a Graells, el 7 de octubre de 1896, instándole a que lo antes posible cursara el dictamen sobre el borrador del Convenio (AMNCN; documento 2). El papel de Graells se vislumbraba por tanto trascendental, puesto que además España -a diferencia de otros países- había enviado a la conferencia de 1895 una representación meramente burocrática, como el propio Graells censuró de manera breve pero incisiva: «Menos los nuestros, es de notar que en las demás naciones se han delegado a personas conocidas por naturalistas o agricultores» (AMNCN; documento 20).

No obstante, el proceso se detuvo de forma transitoria llegado a este punto, porque Graells y el CSA tuvieron que ocuparse de elaborar rápidamente el que ha pasado a ser el primer catálogo español de especies protegidas, y que en buena parte fue un apresurado texto legal donde se intentaron corregir las dudas suscitadas por la Ley de 19 de septiembre de 1896, de conservación de los pájaros, promulgada sin el conocimiento previo del CSA (FerreroGarcía, 2012). Debido a esta demora, el presidente del CSA urgió a Graells, el 17 de marzo de 1897, para que redactara el informe, pues el embajador de Francia, el 16 de enero de 1897, había vuelto a insistir sobre la necesidad de que España adoptara una posición frente al proyecto de Convenio (AMNCN; documento 16). Las prisas del Gobierno francés se debían a que el borrador había sido rotundamente rechazado por Holanda, Italia, Reino Unido y Rusia - a pesar de que sus representantes lo habían aceptado en la conferencia de 1895-, por lo que la existencia misma del Convenio corría grave peligro (Herman, 1907).

Por fin, el 26 de marzo de 1897, Graells evacuó el esperado informe al presidente del CSA (AMNCN; documento 15) y, al día siguiente, el secretario general del CSA convocó a Graells a la reunión plenaria de este organismo que se celebraría el día 30 de ese mes, en el Ministerio de Fomento (AMNCN; documento 17), exclusivamente para tratar la ponencia relativa al Convenio. Para confirmar la realización de dicha reunión y conocer su desenlace es preciso buscar en las actas del CSA, en la sesión del 30 de marzo de 1897 (AMA). En esta, bajo la presidencia de F. García Gómez de la Serna, Graells explicó «el alcance y el objeto del proyecto de Convenio y las observaciones que se hacían a 
cada uno de los puntos que el mismo contenía»; además, mostró su disposición a dirigirse personalmente al Gobierno -si ello fuera preciso- para solventar las dudas que pudieran persistir. El CSA, en cualquier caso, aprobó el dictamen.

Queda acreditada, con esto, la labor esencial de Graells y la total confianza que el conjunto del CSA depositó en él. Con seguridad, aparte de su incontestable prestigio, debió pesar su experiencia en este organismo del que había sido nombrado vocal en 1874 (AMA; expediente sin fecha) y al que perteneció hasta el día de su muerte; de hecho, su plaza vacante no se cubrió hasta cuatro meses después, en junio de 1898 (AMA). En este sentido, E. Hernández-Pacheco ya señaló que, con posterioridad a su controvertida destitución como director del Museo Nacional de Ciencias Naturales y del Real Jardín Botánico, en 1867, Graells mantuvo una constante actividad en diversos órganos consultivos ministeriales, donde siempre fue apreciado (Izquierdo \& Martín, 2009).

Una vez solventada esta cuestión, resulta obligado detenerse para valorar el resultado técnico de este complejo proceso administrativo, esto es, el detallado informe o dictamen que Graells firmó, en Madrid, el 26 de marzo de 1897 (AMNCN; documento 15), y que el CSA corroboró con tanta rapidez. Este carácter específicamente pericial nos lo confirma el propio Graells al comienzo del manuscrito, cuando manifiesta la necesidad de estudiar el proyecto de Convenio «bajo dos puntos de vista: uno diplomático, y el otro técnico», para a continuación consignar que «el primero compete al Ministerio de Estado», mientras que para el segundo sí se atribuye la plena competencia.

El dictamen en sí consiste en la exposición de los 15 artículos que componían el borrador (en el Convenio suscrito en 1902 se le añadió uno más), expresando en cada uno de ellos normalmente su total acuerdo aunque, en ocasiones, creía necesario puntualizar frases o mostrar su disconformidad con ciertos aspectos. Así, por ejemplo, Graells entiende que la protección de los huevos, nidos y crías debería ceñirse solo a las aves del anexo 1, es decir, a las declaradas como útiles a la agricultura por su condición de insectívoras, mientras que el proyecto de Convenio -y también el texto finalmente aprobado en 1902- parecía amparar en su artículo 2 a nidos, huevos y polladas de cualquier taxón. En cambio, Graells se muestra en contra de que los propietarios de los edificios pudieran destruir los nidos cons- truidos en ellos por los pájaros (posibilidad que sí permitía expresamente un apartado del citado artículo 2), y lo razona diciendo: «El párrafo $3 .^{\circ}$ es demasiado absoluto, pues consiente la destrucción de aves insectívoras importantísimas, como son las especies todas de golondrinas, aviones y vencejos...». Otro ejemplo llamativo es el comentario al artículo 3, que prohibía el uso de cualquier medio de captura no selectivo. Graells piensa que debería reducirse esta prohibición únicamente a las aves insectívoras, ya que «no existe razón fundada para impedir que a las aves dañinas no se les pueda exterminar del modo más eficaz que se pueda, hasta empleando el veneno, como se hace con las fieras».

De lo expuesto con anterioridad se podría deducir, quizá, que Graells adoptó posturas contradictorias, unas veces sumamente proteccionistas (verbigracia, a favor de todos los componentes de las familias Apodidae e Hirundinidae) y otras, sin embargo, en apariencia muy poco respetuosas con el medio ambiente. Pero se trataría de un punto de vista simplificador, porque Graells en realidad siguió siempre el mismo criterio, el de un utilitarismo extremo, que va incluso más allá del enfoque antropocéntrico que, como se ha indicado al principio, posee innegablemente el Convenio. Esta visión que tenía de la naturaleza útil, de la aplicación práctica de los conocimientos, ha sido descrita en numerosas ocasiones (e.g., Aragón, 2009; Galera, 2009), y es el impulso que subyace en gran parte de su descomunal obra (Martín \& Izquierdo, 2009). No en vano, Graells heredó el entusiasmo por la dimensión aplicada de la ciencia directamente de su principal mentor, el botánico Mariano La Gasca, y de la tradición científica catalana, y esa idea la propaló y reivindicó durante toda su vida (Aragón, 2006).

En definitiva, el objetivo que llevó a Graells a promover la conservación de las aves insectívoras no fue muy distinto al que le incentivó en algunos de sus mejores trabajos entomológicos, como los relacionados con la devastadora plaga de la filoxera de la vid a raíz de su llegada a Europa (PérezMoreno et al., 2009; Torres-Vila et al., 2009); esto es, el afán de ayudar a los labradores obteniendo -y transmitiendo- datos o logros que beneficiasen a los cultivos. Y para ese encomiable fin unas veces estimó necesario la salvaguardia completa de ciertos taxones, y otras encontró justificable el uso de potentes venenos contra los animales que él consideraba enemigos del agro. 
Graells acaba su informe añadiendo varios comentarios. De este modo, subraya que la lista de especies protegidas por el proyecto de Convenio está «en completa armonía con la que nuestro Gobierno ha señalado en la Real Orden del 25 de noviembre de 1896». No podía ser de otra manera, pues dicha norma la había elaborado él mismo precisamente para aclarar y acomodar la Ley de 19 de septiembre de 1896, de protección de los pájaros, al borrador del Convenio (Ferrero-García, 2012). Y de nuevo asoma su percepción utilitarista de las cosas cuando, aun viendo razonable que a las aves de presa se las considere perniciosas, afirma no obstante lo siguiente: «Verdad es, que de tales acusaciones debemos excusar a las vulturideas que solo se alimentan de cadáveres corrompidos, purgando a la atmósfera de las pútridas emanaciones».

Esta frase de Graells esconde, probablemente, más información adicional. Así, en la literatura ornitológica de aquel tiempo, la familia Vulturidae englobaba con frecuencia a todos los buitres ibéricos excepto al quebrantahuesos (Gypaetus barbatus) (Graells, 1853; Degland \& Gerbe, 1867; Reyes, 1886; Arévalo, 1887), y este último taxón aparecía, tanto en el proyecto de Convenio como en el texto que al final se firmó en 1902, en el anexo de especies nocivas. En cambio, los miembros de la familia Vulturidae, que igualmente se incluyeron en este anexo en el proyecto inicial, después desaparecieron del acuerdo definitivo (Herman, 1907). Aunque se trate de una mera especulación, no deja de ser atractiva la hipótesis de que esta opinión de Graells -y tal vez la de otros expertos que, en los diferentes países, se les encomendara la labor de evaluar esta convención- pudiera haber contribuido a redimir a la mayoría de los buitres de su inserción en el comprometido listado de especies perniciosas. Y por otro lado, no debiera extrañar que Graells se abstuviera de defender al quebrantahuesos: incluso J. Arévalo Baca, en su memorable Aves de España, se refería a esta rapaz asegurando que «se alimenta de presas vivas», en concreto de «corderillos y cabritos, por lo que es muy perseguida» (Arévalo, 1887); y desde luego esta creencia se podía encontrar en algunos de los mejores tratados sobre la avifauna europea de la segunda mitad del siglo XIX (e.g., Degland \& Gerbe, 1867).

En cualquier caso, Graells terminó su informe declarando su conformidad con el proyecto de Convenio:

«La Ponencia no titubea en proponer al Consejo, apoye la adherencia de España a la Convención internacional propuesta por el Gobierno de la República francesa. El Consejo sin embargo con su superior criterio, y acuerdo, determinará en este importantísimo asunto, lo que más convenga manifestar al Gobierno»".

Como se ha comentado anteriormente, cuatro días después el CSA se reunió en pleno, escuchó los argumentos de Graells y aprobó el dictamen sin dudarlo. Once meses más tarde fallecía en Madrid a los 89 años. A diferencia de otras ocasiones no pudo ver, por tanto, la trascendencia de su esfuerzo: la firma por parte de España, el 19 de marzo de 1902, del Convenio Internacional para la Protección de las Aves Útiles a la Agricultura.

\section{Agradecimientos}

Al Dr. Luis Miguel Torres Vila, del Servicio de Sanidad Vegetal de la Junta de Extremadura, por sus oportunos comentarios al manuscrito inicial. A Pilar Elvira Vegas, Encarnación Higueras Plaza y Luz Peña Villarroya, por su inestimable ayuda en la traducción del resumen al inglés. Y a M. ${ }^{\mathrm{a}}$ del Carmen de San José Moreno, por su continuo apoyo y ayuda en todo momento.

\section{Referencias}

Aragón, S., 2006. Un relevo generacional en la historia natural española. La Gasca y Graells: del científico liberal al naturalista isabelino. Asclepio. Revista de Historia de la Medicina y de la Ciencia, 58(2): 203230.

Aragón, S., 2009. La institucionalización de la zoología en España. En: E. Cervantes (coord.). El naturalista en su siglo: homenaje a Mariano de la Paz Graells en el CC aniversario de su nacimiento. Instituto de Estudios Riojanos. Logroño: 175-187.

Aragón, S. \& Villena, M., 2010. Les premières tentatives d'institutionnalisation de la zoologie en Espagne. Revue d'histoire des sciences, 63(2): 473-499.

Arévalo, J., 1887. Aves de España (memorias de la Real Academia de Ciencias Exactas, Físicas y Naturales, tomo XI). Imprenta de viuda e hijo de Aguado. Madrid. 474 pp.

Barclay-Smith, P., 1959. The British contribution to bird protection. Ibis, 101(1): 115-122.

Boardman, R., 2006. The international politics of bird conservation: biodiversity, regionalism and global governance. Edward Elgar Publishing. Cheltenham. $265 \mathrm{pp}$.

Bowman, M., Davies, P. \& Redgwel, C., 2010. Lyster's International Wildlife Law. Second Edition. Cambridge University Press. Cambridge. 784 pp. 
Bowman, M. \& Redgwel, C., 1996. International law and the conservation of biological diversity. Kluwer Law International. London. 334 pp.

Casado, S., 2009. Mariano de la Paz Graells, el último naturalista total. Quercus, 276: 30-33.

Cervantes, E., 2009. (coord.). El naturalista en su siglo: homenaje a Mariano de la Paz Graells en el CC aniversario de su nacimiento. Instituto de Estudios Riojanos. Logroño. 280 pp.

Degland, C. D. \& Gerbe, Z., 1867. Ornithologie européenne ou Catalogue descriptif, analytique et raisonné des oiseaux observés en Europe. Deuxième édition. Tome I. J. B. Baillière \& fils. Paris. 607 pp.

Desai, B. H., 2010. Multilateral environmental agreements: Legal status of the secretariats. Cambridge University Press. New York. 330 pp.

Ferrero-García, J. J., 2010. La conservación de las aves en la España de la Restauración. Quercus, 294: 22-29.

Ferrero-García, J. J., 2011. El primer catálogo español de especies protegidas (1896): análisis de su contenido y autoría de Graells. Graellsia, 67(1): 103-107.

Ferrero-García, J. J., 2012. Mariano de la Paz Graells y la protección de la fauna silvestre. Quercus, 312: 3035 .

Galera, A. 2009. Mariano Graells o la naturaleza útil. En: E. Cervantes (coord.). El naturalista en su siglo: homenaje a Mariano de la Paz Graells en el CC aniversario de su nacimiento. Instituto de Estudios Riojanos. Logroño: 241-249.

García-Maceira, A., 1882. Beneficios de las aves insectívoras. Establecimiento tipográfico de Gregorio Juste. Madrid. 26 pp.

Graells, M. P., 1853. Catálogo metódico de las aves observadas hasta el día por el vocal de la Sección Zoológica D. Mariano de la Paz Graells en el área de la Fauna Matritense. En: F. de Lujan (ed.). Memoria que comprende el resumen de los trabajos verificados en el año 1852 por las diferentes secciones de la comisión encargada de formar el mapa geológico de la provincia de Madrid y el general del reino, presentada el 11 de junio de 1853 al Excmo. Señor Ministro de Fomento. Imprenta, fundición y librería de don Eusebio Aguado. Madrid: 73-86.

Graells, M. P., 1882. Los aliados del labrador en su lucha entomológica. Conferencia dada por el Excmo. Señor don Mariano de la Paz Graells en el Jardín Botánico la tarde del día 21 de junio de 1882. Imprenta y litografía de Nicolás González. Madrid. 30 pp.

Herman, O., 1907. The international convention for the protection of birds concluded in 1902; and Hungary. Historical sketch. Victor Hornyánszky, court printer. Budapest. 241 pp.

Herman, O. \& Owen, J. A., 1909. Birds useful and birds harmful. University Press. Manchester. 387 pp.
Housse, R., 1929. Anotaciones sobre el gorrión. Revista Chilena de Historia Natural, 33(1):107-120.

Izquierdo, I. \& Martín, C., 2008. Graells y Agenjo en GRAELLSIA. Graellsia, 64(2): 349-350.

Izquierdo, I. \& Martín, C., 2009. El Museo de Graells. En: E. Cervantes (coord.). El naturalista en su siglo: homenaje a Mariano de la Paz Graells en el CC aniversario de su nacimiento. Instituto de Estudios Riojanos. Logroño: 189-204.

Kiss, A. \& Shelton, D., 2004. International environmental law. Third Edition. Transnational Publishers. Ardseley. 667 pp.

MacPherson, A. H. \& Momber, G. A., 1909. Legislation for the protection of birds. The Royal Society for the Protection of Birds. London. 68 pp.

Martín, C. \& Izquierdo, I., 2009. Memorias, catálogos, prontuarios, manuales... la obra impresa de Mariano de la Paz Graells. En: E. Cervantes (coord.). El naturalista en su siglo: homenaje a Mariano de la Paz Graells en el CC aniversario de su nacimiento. Instituto de Estudios Riojanos. Logroño: 29-58.

Michelet, J., 1858. L'insecte. L. Hachette et C. ${ }^{\text {ie }}$ Paris. 404 pp.

Ministerio de Estado, 1907. Convenio para la protección de los pájaros útiles á la agricultura. Gaceta de Madrid, 185(4 de julio de 1907): 41-42.

Ministerio de Fomento, 1896. Real Orden de 25 de noviembre de 1896, por la que se aprueba un catálogo de las aves cuya caza debe prohibirse en todo tiempo. Gaceta de Madrid, 337(2 de diciembre de 1896): 831-832.

Oustalet, E., 1884. Rapport a M. le Ministre de l'instruction publique et des Beaux-arts sur le congrès et l'exposition ornithologiques de Vienne, en 1884. Burlin \& Cie. Angers. 406 pp.

Oustalet, E., 1893. La protection des oiseaux. Jouvet \&

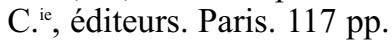

Peña, A., 1905. Tratado de las aves insectívoras cuya caza está prohibida en España. Imprenta de Henrich

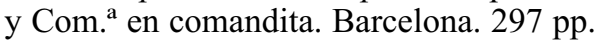

Pérez-Moreno, I., Marco, V., Moreno, F. \& Sáenz de Cabezón, F. J., 2009. Contribución de D. Mariano de la Paz Graells a la entomología española. En: E. Cervantes (coord.). El naturalista en su siglo: homenaje a Mariano de la Paz Graells en el CC aniversario de su nacimiento. Instituto de Estudios Riojanos. Logroño: 155-173.

Rábano, I. \& Aragón, S., 2007. Nuevos datos históricos sobre la Comisión del Mapa Geológico de España. Boletín Geológico y Minero, 118(4): 813-826.

Reyes, V., 1886. Catálogo de las aves de España, Portugal é Islas Baleares. Anales de la Sociedad Española de Historia Natural, 15(1): 5-109.

Sands, P., 1995. Principles of international environmental law I: frameworks, standars and implementation. Manchester University Press. Manchester. 773 pp. 
SEO, 1956. El nuevo convenio internacional para la protección de las aves. Ardeola, 2(2): 241-248.

Torres-Vila, L. M., Ferrero-García, J. J., MartínVertedor, D. \& Sánchez-González, Á., 2009. La sanidad vegetal en Extremadura en el siglo XIX. En: Dioses, mitos y demonios: la agricultura extremeña en el siglo XIX. Colección: historia agraria y rural. Consejería de Agricultura y Desarrollo Rural, Junta de Extremadura. Badajoz: 97-114.

van Heijnsbergen, P., 1997. International legal protection of wild fauna and flora. IOS Press. Amsterdam. $261 \mathrm{pp}$.

Vogt, C. [traducido del alemán por M. G. Bayvet], 1867. Leçons sur les animaux utiles et nuisibles: les bêtes calomniées et mal jugées. C. Reinwald, éditeur. Paris. 334 pp.

\section{ABSTRACT}

Graells's contribution to the Spanish position in the first international convention to protect certain wildlife species (1902)

The process followed by Spain to decide whether it should subscribe the International Convention for the Protection of Birds Useful to Agriculture, signed in 1902 is described. The fundamental role played by Mariano de la Paz Graells, one of the foremost Spanish naturalists of the $19^{\text {th }}$ century, in the technical review and acceptance of the draft agreement, drawn up in 1895, is highlighted. Furthermore, a critical analysis of Graells's position in relation to this so-called first international convention for the conservation of certain wildlife species, has been carried out.

Keywords: Species protection; international conventions; birds; agriculture; $19^{\text {th }}$ century; Mariano de la Paz Graells; Spain.

\section{RESUMEN}

Se describe el proceso que siguió España para decidir si debía suscribir el Convenio Internacional para la Protección de las Aves Útiles a la Agricultura, firmado en 1902. Se pone de manifiesto el papel fundamental que tuvo Mariano de la Paz Graells, uno de los más importantes naturalistas españoles del siglo XIX, en el examen técnico y aceptación del proyecto de dicho convenio, redactado en 1895. Además, se ha realizado un análisis crítico de la postura de Graells en relación con la que puede calificarse como la primera convención internacional para la conservación de ciertas especies de la fauna silvestre.

Palabras clave: Protección de especies; convenios internacionales; aves; agricultura; Mariano de la Paz Graells; España. 